\title{
13 novembre 2015 : terrorisme, résilience, et espoir
}

\author{
November 13, 2015 : Terrorism, Resilience and Hope
}

\author{
B. Riou \\ (C) SFMU et Lavoisier SAS 2016
}

Le 13 novembre 2015, la région parisienne était la cible d'un attentat terroriste multisite sans précédent, en temps de paix, par le nombre de victimes tuées et blessées [1]. Parmi ces victimes, le monde de l'urgence a vu disparaitre l'un des siens, le Dr Stella Verry, médecin du Samu-Centre 15 de Paris, tuée lors de la fusillade du restaurant « Le Petit Cambodge », dont je souhaite ici honorer la mémoire [2].

Face à cet attentat, dont le but était de répandre la terreur, nous avons assisté à une mobilisation, un élan de solidarité et une coopération entre tous les acteurs du système de santé qui a frappé les esprits de tous les observateurs. Cette formidable mobilisation incluant les soignants mais aussi les secouristes, le personnel non soignant des hôpitaux, et les hôpitaux d'instruction des Armées a permis de limiter le nombre de vies perdues et d'offrir à chaque victime blessée des soins de niveau comparable à ceux dont elle aurait bénéficié en dehors d'un afflux massif. Il faut néanmoins reconnaître que ces attentats sont survenus à un moment facilitateur (le vendredi soir) et dans une région très peuplée (environ 12 millions d'habitants) comportant des moyens préhospitaliers et hospitaliers importants sur un espace géographique concentré. Les Annales Françaises de Médecine d'Urgence ont souhaité ouvrir leurs colonnes à une série d'articles de retour d'expérience (RETEX) afin de rendre accessible le maximum d'information sur la façon dont ces événements ont pu être gérés par l'ensemble des acteurs, identifiant ce qui a bien fonctionné, ce qui a parfois dysfonctionné, et surtout ce qui aurait pu dysfonctionner face à un événement d'une telle ampleur. Les éditions Lavoisier et la Société Française de Médecine d'Urgence (SFMU) ont accepté que l'ensemble de ces articles soient disponibles en libre accès sur internet, qu'ils en soient remerciés. J'espère que nos lecteurs y trouveront les informations qui leur per-

\section{B. Riou $(\square)$}

Rédacteur en chef des Annales Françaises

de Médecine d'Urgence. Service d'accueil des urgences,

GH Pitié-Salpêtrière-Charles Foix,

Assistance Publique Hôpitaux de Paris,

Sorbonne Universités, UPMC Univ Paris 06, Paris

47-83 boulevard de l'Hôpital, F-75651 Paris cedex 13

e-mail : bruno.riou@aphp.fr mettront d'actualiser leurs plans en cas d'afflux de nombreuses victimes et de les adapter à leurs considérations locales ou régionales. Plusieurs éléments clés se dégagent de cet ensemble d'articles :

- il convient de se "préparer à être surpris » car aucun plan, aussi bien rédigé soit-il, ne permet de faire face à toutes les éventualités [3] ;

- le recours à l'échelon supra-régional est un élément majeur lorsque la région touchée est susceptible d'être en situation de saturation, signe d'une véritable catastrophe, et ce recours n'a de chance d'être pleinement utile que s'il est suffisamment anticipé ;

- des scénarios doivent être mieux préparés, que ce soit en cas d'afflux de victimes pédiatriques, ce qui aurait pu être le cas de l'attentat du Stade de France, ou si l'hôpital est la cible d'un attentat ;

- lorsque des équipes font face à la saturation, le tri et la chirurgie doivent être adaptés. Ce tri et cette adaptation, bien connus et enseignés par les équipes médicales militaires, et utilisés lors des opérations extérieures, sont souvent méconnus des équipes civiles. Le Service de Santé des Armées (SSA) et les universitaires de chirurgie doivent réfléchir aux moyens de transmettre rapidement aux équipes civiles cette connaissance essentielle.

Lors de la première conférence RETEX des attentats présentée à Paris, Pierre Carli a souligné le rôle essentiel du système de santé dans la résilience de la population française face à ces attentats : "L'action des services de santé $s$ 'oppose directement à l'objectif du terrorisme par la résistance des services à la désorganisation, malgré l'agression, et le maintien de la qualité et de la sécurité des soins malgré l'afflux de victimes et l'effet de surprise. Elle diffuse une image positive, non violente, qui privilégie l'individu, se substitue à l'engrenage de l'agression et de la répression, soigne les victimes (mais aussi les terroristes), et incite le public à s'impliquer pour sauver les victimes ${ }^{*}$. C'est un

\footnotetext{
*Carli P. Télion C. La réponse médicale aux attaques terroristes simultanées sur plusieurs sites à Paris, Séminaire des Services d'Urgences, Campus Erasme, Bruxelles, Belgique, 12 décembre 2015
} 
point remarquable qui montre que notre système de santé reste un pilier de notre société, malgré des difficultés financières et des mutations profondes. Les structures d'urgences ont montré, encore une fois, leur capacité à faire face et à défendre les valeurs du juste soin [4], au quotidien comme dans des situations exceptionnelles.

Cette date du 13 novembre 2015 marquera durablement les esprits des citoyens français. Toutefois, le hasard des publications au Journal Officiel fait qu'il s'agit aussi de la date de création du diplôme d'études spécialisées (DES) de médecine d'urgence [5], officialisant la reconnaissance de la médecine d'urgence en France comme spécialité à part entière. Comme si les Ministères concernés avaient pris conscience, enfin, de la nécessité de cette reconnaissance, déjà obtenue de longue date dans la plupart des pays européens [6]. Face à la grande Histoire, la date du 13 novembre 2015 est aussi une date remarquable pour la petite histoire de la médecine d'urgence en France et une note d'espoir pour les médecins urgentistes d'aujourd'hui et de demain.

\section{Références}

1. Hirsch M, Carli P, Nizard R, et al (2015) The medical response to multisite terrorist attacks in Paris. Lancet 368:2535-8

2. Vies de médecins. Dr Stella Verry : in memoriam (2016). Le Quotidien du médecin $\mathrm{N}^{\circ} 9451$. http://www.lequotidiendumedecin.fr/ actualites/portrait/2015/11/19/dr-stella-verry-memoriam_781646 (Dernier accès le 5 janvier 2016)

3. Lagadec P (2015) Le continent des imprévus. Paris: Manitoba / Les Belles Lettres

4. Samu-urgences de France (2016) Livre blanc. Organisation de la médecine d'urgence en France : un défi pour l'avenir. http://www. samu-de-france.fr/documents/actus/129/821/livre-blanc-sudf-151015. pdf (Dernier accès le 5 janvier 2016)

5. République Française (2015) Arrêté du 13 novembre 2015 fixant la liste des diplômes d'études spécialisées de médecine. JORF n0281 du 4 décembre 2015. http://www.legifrance.gouv.fr/eli/ arrete/2015/11/13/MENS1525635A/jo/texte (Dernier accès le 5 décembre 2015)

6. Nemitz B, Carli P, Carpentier F, et al (2012) Référentiel métiercompétences pour la spécialité de médecine d'urgence. Ann Fr Med Urg 2:125-38 